\title{
Diagnosis and management of pseudomembranous candidiasis
}

\begin{abstract}
The patient risk for opportunistic infection, especially fungal infection. Candidiasis is one of the most frequent fungal infections determined in these immunosuppressive patients and its epidemiology has changed over the last two decades. Pseudomembranous candidiasis (oral thrush) presents as creamy white lesions on the oral mucosa and a diagnostic feature of this infection is that these plaques can be removed by gentle scraping leaving behind an underlying erythematous mucosal surface. The most effective agents used in the treatment of Candida species are the antifungal agents belonging to polyene and azole groups. Here we report a case of "Oral thrush on the Palate \& Tongue" in a 65year old female patient.
\end{abstract}

Keywords: oral candidiasis, palate, tongue, koh staining, candida albicans, metered dose inhaler
Volume 8 Issue 3 - 2017

\author{
Samiksha Acharya,' Vidya K Lohe, ${ }^{2}$ Rahul R \\ Bhowate $^{2}$ \\ 'Department of Oral Medicine Diagnosis and Radiology Guru \\ Gobind Singh College of Dental Sciences and Research Centre, \\ India \\ ${ }^{2}$ Depatment of Oral Medicine Radiology Sharad Pawar Dental \\ College Hospital DMIMS, India
}

\section{Correspondence: Samiksha Acharya Senior lecturer Department of Oral Medicine Diagnosis and Radiology Guru Gobind Singh College of Dental Sciences and Research Centre Burhanpur Madhya Pradesh 45033I, India, Tel 9503279165, Emailsmkacharya@gmail.com}

Received: January 26, 2017 | Published: August 23, 2017
Abbreviations: HIV, human immunodeficiency virus; AIDS, acquired immune deficiency syndrome; MDI, metered dose inhaler; GMS, gomori methenamine silver; PAS, periodic acid Schiff

\section{Introduction}

Candidiasis is a common opportunistic oral Candida infection that develops in the presence of one of several predisposing conditions. ${ }^{1,2}$ The term Candida originates from the Latin word candid, meaning white. The spores of Candida are a commensal, harmless form of a dimorphic fungus that becomes invasive and pathogenic pseudohyphae when there is a disturbance in the balance of flora or in debilitation of the host. ${ }^{3,4}$ The most common species being Candida albicans and the other species include Candida tropicalis, Candida glabrata, Candida pseudotropicalis, Candida guilliermondii, Candida parapsilosis, and Candida krusei. ${ }^{5}$ Oral candidiasis is also known as oral candidosis, oral thrush, moniliasis, oral mycosis, oral yeast infection, or Candidal stomatitis. Candidal species are relatively common inhabitant of the oral cavity, gastrointestinal tract, and vagina of clinically normal persons. Candidiasis is caused by an overgrowth of the superficial fungus Candida albicans (C. albicans) ${ }^{6,7}$ C. albicans is a commensal organism residing in the oral cavity in a majority of healthy persons. ${ }^{1,2}$ Oral candidiasis is one of the most common, treatable oral mucosal infections seen in persons with human immunodeficiency virus (HIV) infection or acquired immune deficiency syndrome (AIDS). Oral candidiasis can be a frequent and significant source of oral discomfort, pain, loss of taste, and aversion to food. ${ }^{3,8}$

Pseudomembranous candidiasis (thrush) is the most common form of candidiasis. ${ }^{1,2}$ Thrush forms soft, friable, and creamy plaques on the mucosa that can be wiped off, leaving a red, raw or bleeding, and painful surface. The buccal mucosa, palate, and tongue are common location. ${ }^{2}$ The lesions may involve the entire oral mucosa or relatively localized areas where normal cleansing mechanisms are poor., ${ }^{2,9}$ The pseudomembrane consists of a network of candidal hyphae containing desquamated cells, microorganisms, fibrin, inflammatory cells, and debris. ${ }^{2}$ Diagnosis of thrush is usually based on clinical criteria. Direct smear microscopic examination with potassium hydroxide and culture are helpful. ${ }^{10}$ The management of oral thrush includes topical antifungal applications containing the polyene drugs like Nystatin and Amphotericin or azole group drugs like Clotrimazole, Fluconazole, and Ketoconazole. ${ }^{6}$ The purpose of this case to discuss about the diagnosis investigation and management of pseudomembranous candidiasis.

\section{Case report}

A 65-year-old female patient visited the Department of Oral Medicine and Radiology with the chief complaint of itching and burning sensation in her mouth since one and a half month. Burning sensation increased on taking spicy food. A medical history revealed that she was hypertensive and was asthmatic since 7years. She was treated for asthma with bronchodilator (Salbutamol with Ipratropium bromide) as a reliever. Because of increased frequencies of asthmatic exacerbations her physician prescribed (ICS beclomethasone $400 \mu \mathrm{g}$ ) which the patient was using for about 3 months. On intraoral examination, diffuse curdy white patches were seen on her tongue, right and left buccal vestibules and mucosa, as well as on her hard palate and soft palatal region with diffuse erythema over the soft palate, uvula, and, with scrapable white patches (Figures $1 \& 2$ ). .The complete blood examination \& Randam blood Sugar report showed normal reports. On histopathological evaluation, the smear was made from scrapings of these lesions from the palate and tongues separately and sent to the department of Oral Pathology \& Microbiology. For cytological evaluation, $\mathrm{KOH}$ Staining and $\mathrm{H} \& \mathrm{E}$ staining were done which shows fungal elements. Culture Candida using a Sabouraud's dextrose agar was also done to aid the definitive identification of the fungal organism. Histopathological report confirms the diagnosis as pseudomembranous candidiasis. Patient was advised for CANDID mouth paint twice a day for 15 days. And patient was recalled after 15 days. So, there was a complete remission of the lesion on the day of call.

The lesions were scrapable and left diffuse erythematous areas and bleeds on scraping. Smear made from scrapings of lesions were sent for cytological examination which confirmed the presence of candidal hyphae (Figure 3). 


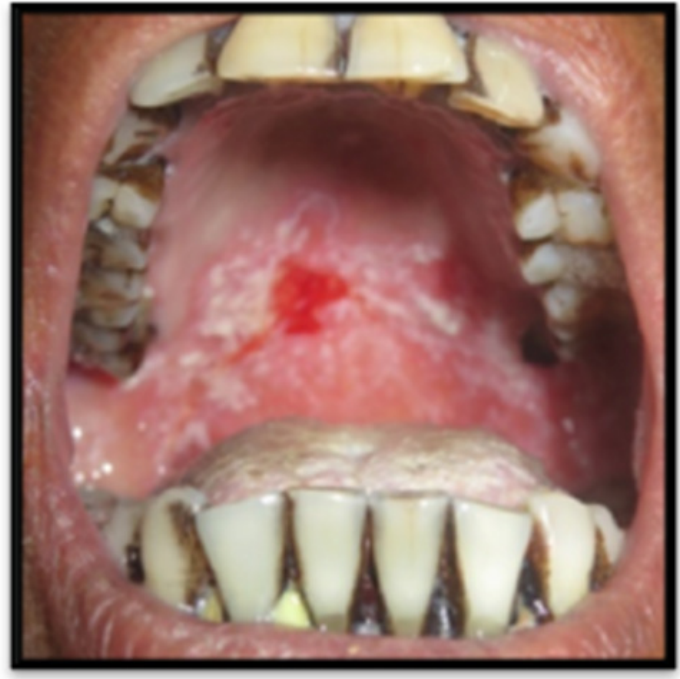

Figure I Shows the erythematous region in the palate.

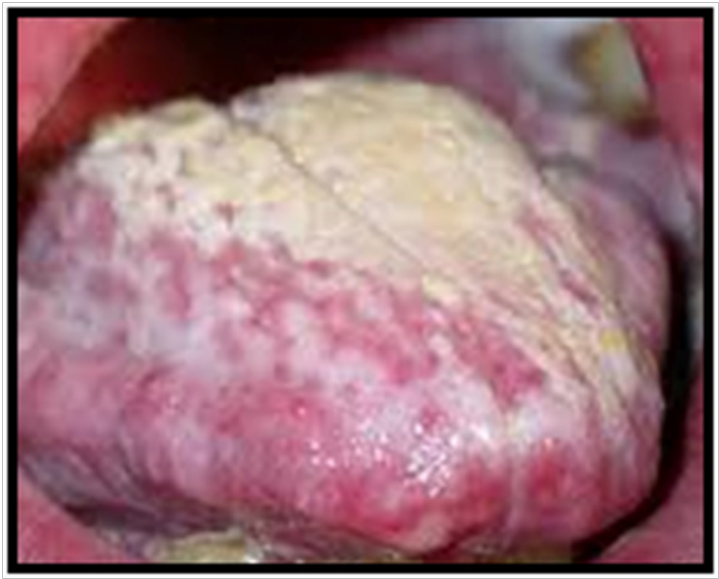

Figure 2 Shows curdy white coat on the tongue.

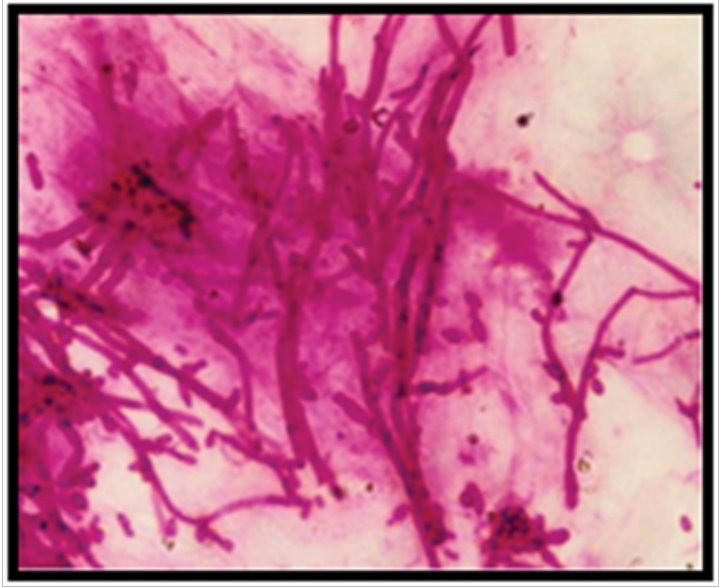

Figure 3 Photomicrograph demonstrates tubular-appearing fungal hyphae and ovoid yeasts of Candida albicans(Periodic acid-schiff stain).

To rule out any underlying systemic cause's complete hemogram and rapid test for HIV was done which revealed all values within the normal range and nonreactive status for HIV. Based on history, clinical presentation and cytological report, final diagnosis of drug- induced pseudomembranous candidiasis was made. The patient was advised to follow strict oral hygiene measures and he was also asked to use spacer along with metered dose inhaler (MDI) while using steroid inhaler with topical application of clotrimazole $1 \%$ mouth paint around 4-5times per day for about 2 weeks. The patient was reviewed after 15 days where he presented with complete remission of the lesions (Figures $4 \& 5$ ).
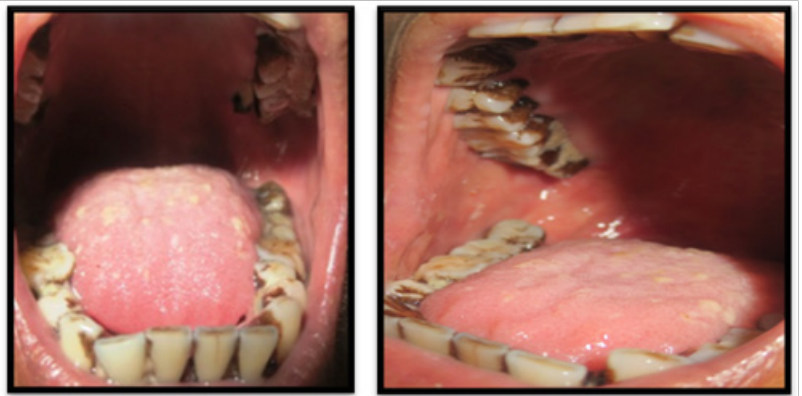

Figure 4 \& 5 Shows Post-treatment view of tongue, hard and soft palate and buccal mucosa.

\section{Discussion}

The term Candida originates from the Latin word candid, meaning white. The spores of Candida are a commensal, harmless form of a dimorphic fungus that becomes invasive and pathogenic pseudohyphae when there is a disturbance in the balance of flora or in debilitation of the host. Candidiasis or oral candidiasis is one of the most common human opportunistic fungal infections of the oral cavity. The incidence of fungal infections has been increasing over the last decades, being more prevalent in developed countries. An increase incidence of the infections is associated with some predisposing factors as the use of dentures, xerostomia, prolonged therapy with antibiotics, local trauma, malnutrition, endocrine disorders, increased longevity of people, among other states that diminish the quality of defense of the individual. ${ }^{3,11}$ The diagnosis can be confirmed microbiologically either by staining a smear from the affected area with periodic acid Schiff (PAS) stain, Gridley stain, or Gomori methenamine silver (GMS) stain or by culturing a swab from an oral rinse. ${ }^{2}$ Culture Candida using a Sabouraud's agar slant was done to aid in the definitive identification of the fungal organism.

This case was diagnosed based on clinical features, which characterized of thrush that is white creamy pseudomembrane (patch) that can be wiped off and leaving erythematous base. Diagnosis of this case was confirmed with microbiology examination through swab and culture using Sabouraud's agar. Candida colony was found in microbiology examination. In older individuals, acute pseudomembranous candidosis often occurs when there is a nutritional limitation, local immune suppression (e.g. steroid inhaler administration for the treatment of asthma), or an underlying disease most notably HIV infection and AIDS., ${ }^{2,12}$ Bronchial asthma is a chronic inflammatory disease of the respiratory system which is characterized by dyspnea, shortness of breath, coughing, and wheezing due to the narrowing of the bronchial airways by muscle spasm, mucosal swelling or nasal and bronchial secretions. An immune complex allergic reaction is suggested to be the etiological factor. ${ }^{13}$ Bronchial asthma itself will not cause any oral lesions but indirect effects of asthma drug therapy can induce clinical lesions. Patients most prone to develop oral manifestations are chronic asthmatics who use corticosteroid inhalants since these are the main stay therapeutic agents in the management of bronchial asthma. ${ }^{6,14}$ Repeated contact 
of steroid inhalant on the oral mucosa can result in the development of acute pseudomembranous candidiasis (oral thrush) because of fungal overgrowth in an area of localized Immunosuppression. ${ }^{6,14}$ According to Salzman et al., ${ }^{15}$ increased concentration of glucose in saliva resulting from the effect of deposited corticosteroids may be responsible for oral candidiasis. ${ }^{6,15}$ A study done in 2013 showed that a relative risk is the highest in the first 3 months of ICS usage, but remain increased up to at least 1 year after ICS initiation..$^{16}$ This steroid-induced infection consists of $\mathrm{C}$. albicans colonies that appear as curdy white lesions located commonly on the soft palate and oropharynx. Eventually, the white precipitates peel off leaving behind an intensely erythematous and raw-looking area.

In this case report, a proper history and clinical evaluation, patient was also analysed for underlying systemic causes. Once the final diagnosis was arrived, appropriate treatment was considered by the use of spacer along with metered dose steroid inhaler and topical application of antifungal agent clotrimazole 1\% mouth paint for 2 weeks. It was effective enough in healing the lesions. Measures like rinsing the mouth with water after MDI use can also prevent the occurrence of oral candidiasis. When the patient was re-evaluated during follow-up visit, he presented with complete remission of the lesions. Topical agents include nystatin suspension and clotrimazole troches, which should be allowed to dissolve slowly in the mouth five times daily for 14days. Patients should avoid eating or drinking for 20 minutes after using clotrimazole troches. Intraoral appliances should be removed during the treatment as the medication works topically and must be in contact with the tissue. Systemic prescription antifungal agents include ketoconazole, fluconazole, and itraconazol. Recent developments for many years, amphotericin B deoxycholate remained the mainstay of treatment for IFIs. The major limitations of its usage are the substantial adverse effects such as fever, chills, nausea and vomiting, electrolyte abnormalities and, most importantly, nephrotoxicity. ${ }^{8}$

\section{Conclusion}

The conclusion of this case is thrush developed due to 3 factors are reduced of host's immune status that was affected by patient's age, oral mucosa environment that contributed to candidiasis i.e. poor oral hygiene, and the present of C. albicans which is normal oral flora. In this case, thrush was diagnosed through clinical feature which was confirmed with microbiology examination and treated with topical antifungals (nystatin oral suspension and miconazole oral gel) which were combined with systemic antifungal (ketoconazole). Diagnosing oral fungal lesions along with appropriate management measures are the prime responsibilities. Carefully recording the medical history is important in identifying this clinical problem. Predisposing factors should be treated or eliminated where feasible. Since topical anticandidal therapy is efficacious in the management of oropharyngeal candidiasis, it alone is not sufficient in asthmatic patients who continue to use steroid inhalers. The prognosis is good for oral candidiasis with appropriate and effective treatment.

\section{Acknowledgments}

None.

\section{Conflicts of interest}

Author declares there are no conflicts of interest.

\section{Funding}

None.

\section{References}

1. Regezi JA, Sciubba JJ, Jordan RCK. Oral pathology clinical pathologic correlations. (4th edn), Saunders, St. Louis, USA. 2003. p.100-104.

2. Nurdiana, Jusri M. Pseudomembranous candidiasis in patient wearing full denture. Dental Journal. 2009;42(2):60-64.

3. Verma A, Kumar P, Kaur L, et al. Oral thrush on the palate \& tongue. International. Journal of Research in dentistry. 2015;5(4):102-106.

4. Byadarahally Raju S, Rajappa S. Isolation and Identification of Candida from the Oral Cavity. ISRN Dent. 2011;2011:487921.

5. Akpan A, Morgan R. Oral candidiasis. Postgrad Med J. 2002;78(922):455-459.

6. Pati A, Susmitha HR, Basappa S, et al. Drug-induced Oral Candidiasis: A Case Report. IJSS Case Reports \& Reviews. 2016;2(12):1-4.

7. Langlais RP, Miller CS. Color atlas of common oral diseases. (3rd edn), Lippincott Williams and Wilkins, Philadelphia, USA. 2003. p.130-131.

8. Yuvraj Singh Dangi, Murari Lal Soni, Kamta Prasad Namdeo. Oral Candidiasis: A Review. Int J Pharm Sci. 2010;2(4):36-41.

9. Bhattacharyya I, Cohen DM, Silverman S. Red and white lesions of the oral mucosa. In: Greenberg MS, Glick M. Burket's oral medicine diagnosis and treatment. (10th edn). Hamilton: BC Decker, Germany. 2003. p.94-101.

10. Laskaris G. Treatment of oral diseases. A concise textbook. (1st edn). Thieme, Stuttgart, Germany. 2005. p.30-32.

11. Carla Garcia-Cuesta, Maria-Gracia Sarrion-Pérez, Bagán JV. Current treatment of oral candidiasis: A literature review. J Clin Exp Dent . 2014;6(5):576-582.

12. Laskaris G. Treatment of oral diseases. A concise textbook. (1st edn), Thieme, Stuttgart, Germany. 2005. p.30-32.

13. Onyedum C, Ukwaja K, Desalu O, et al. Challenges in the management of bronchial asthma among adults in Nigeria: A systematic review. Ann Med Health Sci Res. 2013;3(3):324-329.

14. Fukushima C, Matsuse H, Saeki S, et al. Salivary IgA and oral candidiasis in asthmatic patients treated with inhaled corticosteroid. $J$ Asthma. 2005;42(7):601-604.

15. Salzman GA, Pyszczynski D. Oropharyngeal candidiasis in patients treated with beclomethasone dipropionate delivered by metereddose inhaler alone and with Aerochamber. J Allergy Clin Immunol. 1988;81(2):424-428.

16. Van Boven JF, de Jong-van den Berg LT, Vegter S. Inhaled corticosteroids and the occurrence of oral candidiasis: A prescription sequence symmetry analysis. Drug Saf. 2013;36(4):231-236. 